\title{
Geometric Characterization of Three-Dimensional (3D) Woven Jute Fiber Preforms for Composites
}

\author{
Nesrin Şahbaz Karaduman ${ }^{1 * i[}$ \\ ${ }^{1}$ Department of Textile, Footwear and Leather, Akdagmadeni Vocational High School, Yozgat Bozok University, \\ 66300, Yozgat, Turkey \\ *nesrin38@gmail.com \\ *Orcid: 0000-0001-9555-7044
}

Received: 15 November 2020

Accepted: 30 March 2021

DOI: $10.18466 /$ cbayarfbe. 826090

\begin{abstract}
Fiber-reinforced composite materials have many advantages in various engineering applications when compared to traditional materials such as glass, metals, ceramics, and unreinforced plastics. Recently, textile-reinforced composites are increasingly used in various industries including aerospace, construction, and automotive. This study aims to investigate the geometric characteristics of threedimensional (3D) woven preforms which are used as reinforcement materials in composites. To this end, 3D woven preforms with three different weave types were produced namely 3D orthogonal, 3D plain z-orthogonal, and 3D satin z-orthogonal. The effect of weave pattern and the number of layers on the geometric characteristics of the produced fabrics was investigated. For this purpose, yarn-yarn distances and density, yarn lengths, and yarn angles were measured. The effect of the number of layers on the geometric parameters was limited. Yarn-to-yarn distances in plain-weave fabrics were found to be greater when compared to other types of fabrics whereas the yarn density decreased in plain woven fabrics due to a large number of interlacements. This shows that in composite form, the fiber volume fraction in the filling and $\mathrm{z}$-directions will be lower in the semi-interlaced fabrics when compared to the non-interlaced orthogonal structures. It was also shown that filling and warp angles are a function of weave type while $\mathrm{z}$ yarn angle is associated with the weaving operations such as beat-up, multilayer filling insertion, and warp yarn let off.
\end{abstract}

Keywords: Geometric characterization, Jute fiber, Natural fiber composites, Three dimensional (3D) woven preform.

\section{Introduction}

Fiber-reinforced composites have many advantages over traditional materials such as metals, glass, ceramics, and plastics. These advantages include high strength and stiffness, low weight, tailorability, fracture toughness, and corrosion resistance. Fibers are the main load-bearing element in a composite system due to their high strength and stiffness whereas the matrix determines the final shape of the composite and protects the fibers against harmful environments. The matrix phase is also responsible for homogenously dispersing applied loads to reinforcing fibers. Fiber and matrix type, fiber orientation/architecture, and fiber volume fraction determine the mechanical properties of a composite material.
Textile-reinforced composites are a group of materials that use a textile form as reinforcement such as yarns, woven, knitted, braided, nonwoven, and multiaxial fabrics. Textile fabrics can be manufactured in twodimensional (2D) and three-dimensional (3D) forms. The main difference between the two is the use of through-the-thickness or z-yarns in the latter. The type and parameters of textile structure determine the fiber architecture and orientation as well as fiber volume fraction in composite materials.

The first application of textile fabrics was in the aerospace industry where carbon-carbon composites were produced using layered $2 \mathrm{D}$ textile fabrics. These composites were produced by simply stacking 2D woven fabrics one on top of the other and impregnating with the resin using one of the composite manufacturing methods. These composite structures were prone to 
delamination and showed poor damage tolerance due to a lack of through-the-thickness reinforcement (z-yarns). The solution to these problems of layered structures was the development of 3D fabric manufacturing processes which incorporated z-yarns in the thickness direction to bind the fabric layers and create a compact 3D structure which has good interlaminar strength and damage tolerance [1]. Moreover, 3D fabrics eliminate the laborintensive layering step in composite processing reducing the overall cost and allow net-shape manufacturing. For all these reasons, 3D textile-reinforced composite structures have been used in the aerospace industry since the 1960s. Nowadays 3D textile composites are used in various industries including aerospace, civil engineering, automotive, and sports [2].

In recent years, natural fibers have attracted considerable attention as composite reinforcement due to increased environmental concerns such as global warming and environmental pollution as well as economic reasons such as rising petroleum prices and depletion of fossil fuels. Natural bast fibers such as jute, hemp, flax, and kenaf are increasingly being used as reinforcement materials due to their low density and high strength/stiffness. Natural fibers are environmentally friendly, sustainable, renewable, and biodegradable [3-7]. Owing to these distinct advantages, natural fibers can compete with glass fibers in various composite applications [8-11].

A literature survey showed that the number of studies on the geometric parameters of 3D woven natural fiber fabrics is limited. This study aims to investigate the geometric characteristics of 3D woven jute preforms as a potential candidate as reinforcement in composites. For this purpose, non-interlaced and semi-interlaced 3D woven preforms with three different weave types were produced namely 3D orthogonal, 3D plain z-orthogonal, and 3D satin z-orthogonal. The effect of weave pattern and the number of layers on the geometric characteristics of the produced fabrics was investigated. For this purpose, yarn-yarn distances and density, yarn lengths, and yarn angles were measured. The results were evaluated taking into account the corresponding composite properties.

\section{Materials and Methods \\ 2.1. Materials Used}

$3 \mathrm{D}$ weaving requires three yarn sets such as warp, filling and, z-yarns (through-the-thickness yarns). In this study jute yarns with a linear density of 250 tex were used as warp, filling and z-yarns to make 3D woven preforms. Jute yarns were provided by Erkollar Ltd. (Gaziantep, Turkey).

\subsection{D Woven Fabric Production}

An in-house developed 3D weaving apparatus was used to produce the 3D woven jute fabrics. Two main groups of fabrics were produced such as a) Non-interlaced orthogonal and b) Semi-interlaced orthogonal fabrics. In non-interlaced fabrics, warp, filling, and z-yarns run along the length (x-axis), width (y-axis) and thickness (z-axis) of the fabric respectively without making interlacements with other yarn sets (Figure 1(a) and 2(a)). The production of this fabric requires six distinct steps such as warp let-off, 2D shedding, multilayer filling insertion, z-yarn insertion, beating, and fabric take-up [12]. Figure 3 shows 3D orthogonal weaving principle schematically. The second group of 3D fabrics produced in this study i.e. semi-interlaced fabrics were produced using two distinct weave patterns such as plain-weave and satin-weave. These patterns were chosen due to the fact that plain-weave fabrics contain a large number of interlacements when compared to satin-weave making it easier to observe the effect of any geometrical differences on fabric properties. In these fabrics, warp and filling yarns make interlacements according to 1/1 plain (Figure 1(b), 2(b)) and 1/4 satin (Figure 1(c), 2(c)) weave patterns while $\mathrm{z}$ yarns run along the thickness of the fabrics between adjacent warp yarns without making any interlacements with other two yarn sets. Fabric production started with warp let-off. Then, z-yarns are divided into two groups, such that an odd number of yarns are laid in the $+z$ direction while an even number of yarns are laid in $-z$ direction. Then the filling yarn makes interlacements with warp yarns according to the weave pattern in every layer. Then z-yarns move in alternate directions to their first position such that an odd number of yarns are laid in the $-z$ direction while an even number of yarns are laid in $+z$ direction. This cycle of movements was repeated to make preforms. Two different number of layers such as 2 and 4 layers were used for the production of all the fabrics in this study. Table 1 lists the produced 3D fabric samples together with their sample codes.

Table 1. Produced 3D fabric samples.

\begin{tabular}{llll}
\hline Fabric Type & $\begin{array}{l}\text { Number } \\
\text { of Layers }\end{array}$ & Sample Code \\
\hline Orthogonal & 2 & 3DWO \\
& 4 & 3 3DWP-ZO \\
\hline \multirow{2}{*}{$\begin{array}{l}\text { Semi- } \\
\text { interlaced }\end{array}$} & Plain & 2 & 4 \\
\cline { 2 - 4 } & Satin & 2 & 3DWS-ZO \\
\hline
\end{tabular}


Celal Bayar University Journal of Science

Volume17, Issue 2, 2021, p 121-128

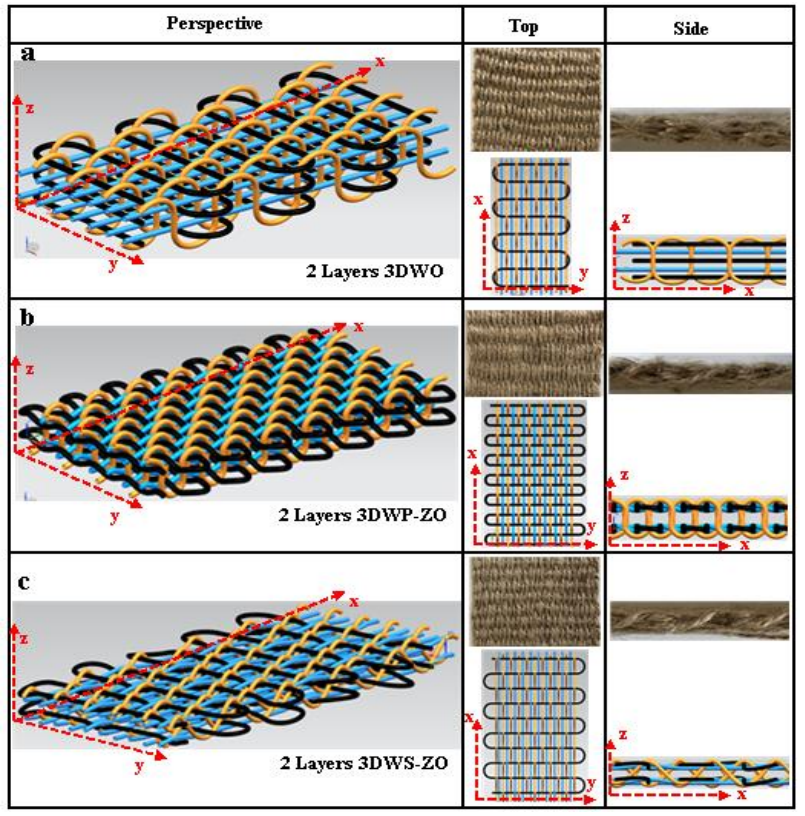

Figure 1. Two-layer fabric samples and their computer drawings (a) 3D orthogonal fabric; (b) 3D plain-weave z-orthogonal fabric; (c) 3D satin-weave z-orthogonal fabric.

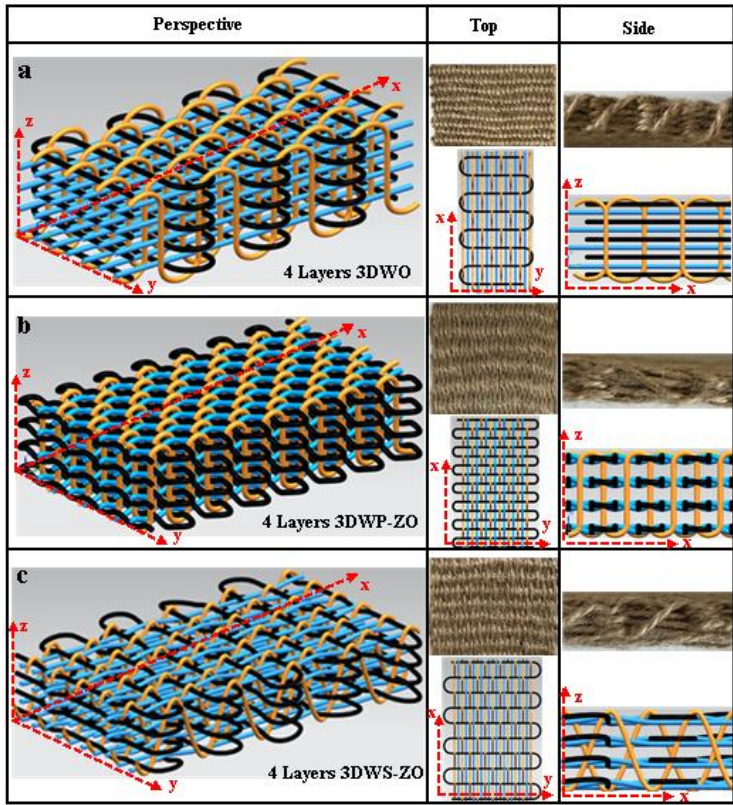

Figure 2. Four-layer fabric samples and their computer drawings (a) 3D orthogonal fabric; (b) 3D plain-weave z-orthogonal fabric; (c) 3D satinweave z-orthogonal fabric.

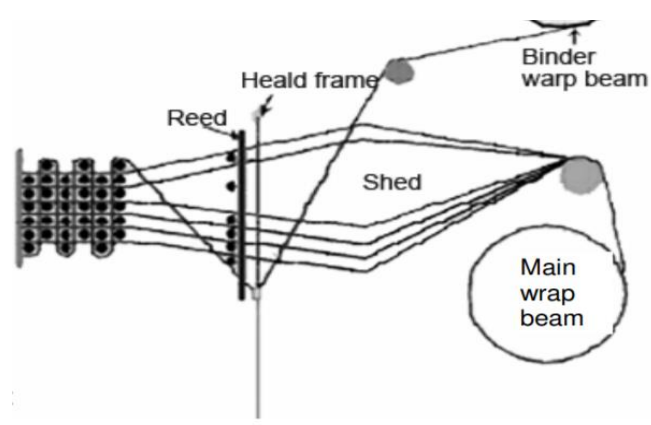

Figure 3. 3D orthogonal weaving principle [13].

\subsection{Computer Modeling}

The produced fabric samples were modeled using NXUnigraphics 7.5 software to clearly demonstrate the yarn paths and interlacements in 3D structures and for future analysis.

\subsection{Determination of Fabric Dimensions}

In 3D woven fabrics, fabric length $(S l)$, width $(S w)$, and thickness $(S t)$ were measured. The fabric length was limited to $20 \mathrm{~cm}$ in all the fabrics. Figure 4 shows the fabric dimensions and yarn sets on actual fabric and

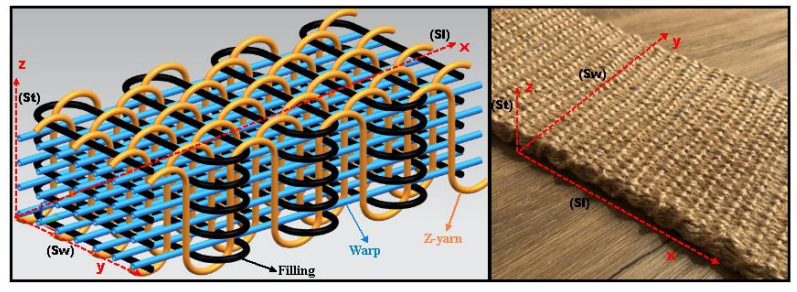

Figure 4. Demonstration of the fabric dimensions and yarn sets on computer model and actual fabric sample. 


\subsection{Determination of Yarn-to-Yarn Distances and Yarn Density}

Yarn-to-yarn distances of adjacent yarns were measured on the 3D woven fabric samples. Warp-warp (w-w), filling-filling (f-f), and z-yarn-z-yarn (z-z) distances were measured on top side ( $x-y$ plane), side ( $x-z$ plane), and cross-section ( $\mathrm{y}-\mathrm{z}$ plane). Figure 5 highlights the yarn-to-yarn distances and other related parameters for measurements.

Yarn density measurements were carried out for warp, filling, and z-yarns on top, side, and cross-section planes of the fabrics. The number of yarns in $5 \mathrm{~cm}$ was considered for yarn density measurements.

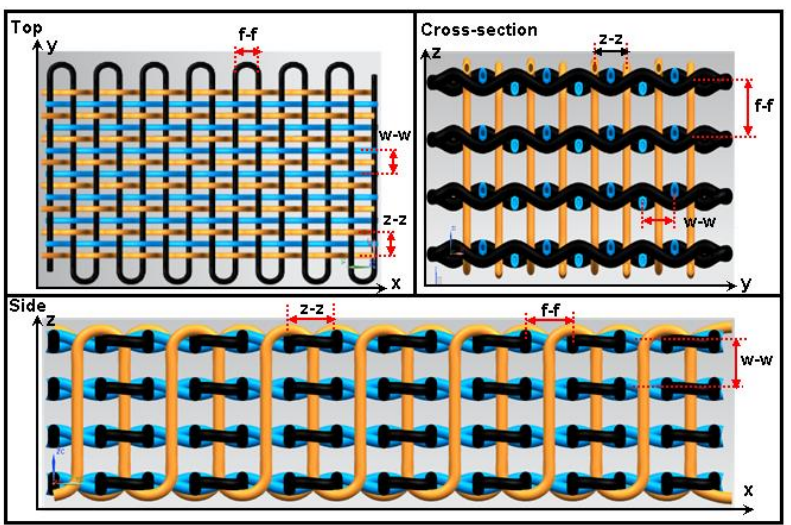

Figure 5. The illustration of the parameters considered in yarn-to-yarn distance measurements.

\subsection{Determination of the Yarn Lengths}

Uncrimped yarn lengths were measured in 3D woven fabrics. In fabric structure, especially at the interlacement points, warp, filling, and z-yarns follow curved paths and the yarns become curved. This is referred to as the "yarn crimp". The length of the path that warp, filling, and z-yarns follow in the fabric structure is hence called the "uncrimped yarn length". In this study, guide yarns were used in order to be able to measure the uncrimped yarn lengths. First, the guide yarn was inserted in the structure following the exact same yarn path as the yarn (i.e. warp, filling or z-yarn) whose uncrimped length is to be measured. Then the ends of this guide yarn were marked before it is withdrawn from the structure.

The distance between the markings on the straightened guide yarn gives the uncrimped yarn length. Uncrimped warp yarn length $(l w)$, uncrimped filling yarn length $(l f)$, and uncrimped z-yarn length $(l z)$ were measured for all fabric types (Figure 6).

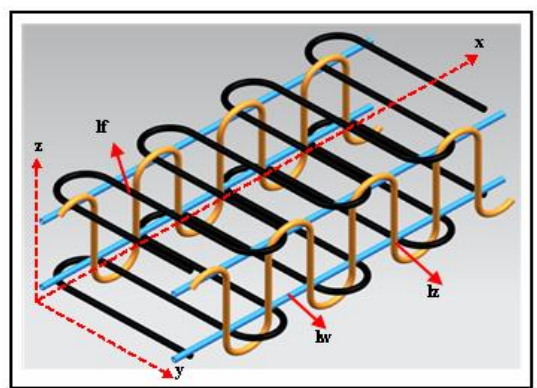

Figure 6. Illustration of uncrimped warp, filing, and zyarn length on the computer model.

\subsection{Determination of the Yarn Angles}

The following yarn angle measurements were carried out in 3D woven fabric structures:

a) The filling angle between the filling yarn and warp yarn in the fabric width direction (y-axis) $(\theta f)$ (Figure 7(a))

b) The filling trajectory angle between the filling yarn and $\mathrm{z}$ - yarn in the fabric thickness direction ( $\mathrm{z}$-axis) $(\theta f z)$ (Figure 7(a))

c) The warp angle between the warp yarn and filling yarn in the fabric length direction (x-axis) $(\theta w)$ (Figure 7(b))

d) The warp trajectory angle between the warp yarn and $\mathrm{z}$ - yarn in the fabric thickness direction (z-axis) $(\theta w z)$ (Figure 7(b))

e) The z-yarn angle between the z-yarn and warp yarn in the fabric length direction (x-axis) $(\theta z)$ (Figure 8)

f) The z-yarn trajectory angle between z-yarn and warp yarn in the thickness direction (z-axis) $(\theta z w)$ (Figure 8)

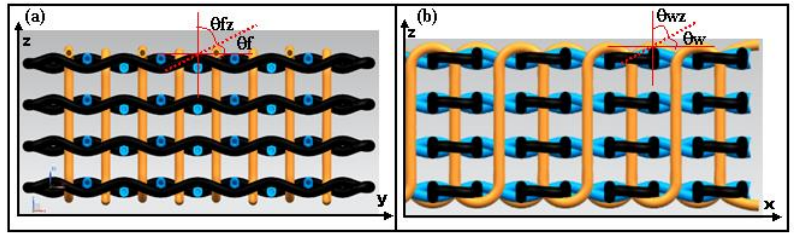

Figure 7. Demonstration of (a) $\theta \mathrm{f}$ and $\theta \mathrm{fz}$ angles (b) $\theta \mathrm{w}$ and $\theta \mathrm{wz}$ angles.

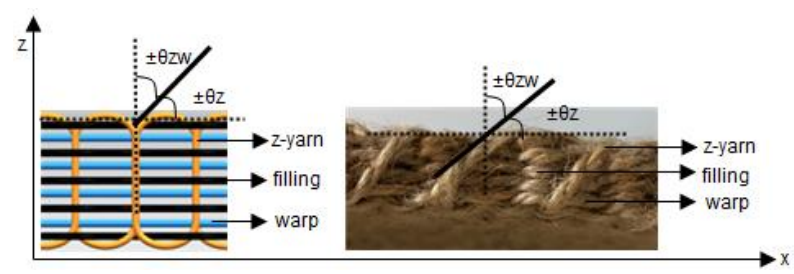

Figure 8. Demonstration of $\theta z$ and $\theta z w$ angles. 


\section{Results and Discussion}

In the current study, the geometric characterization of 3D woven jute fabrics was carried out for their possible application in fiber-reinforced composites. For this purpose, 3D orthogonal, 3D plain z-orthogonal, and 3D satin z-orthogonal woven fabrics were produced (Figure 9).

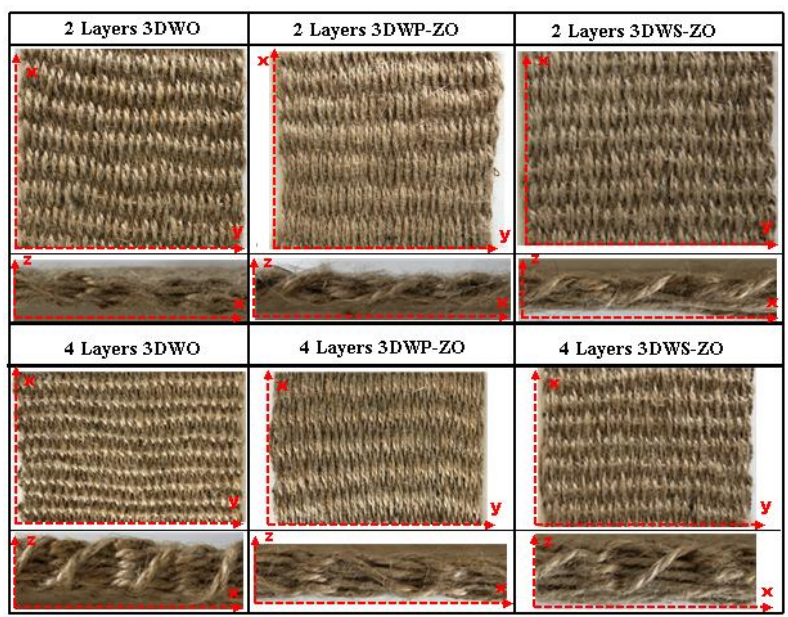

Figure 9. Fabric types produced in the current study.

\subsection{Yarn-to-Yarn Distances and Yarn Density}

Figure 10 shows the relation between the fabric type and the yarn-to-yarn distances measured on the fabric side (xz-plane). Filling-filling and z-yarn-z-yarn distances were found to be the same with each other and greater than the warp-warp distance. The number of layers did not have a significant effect on the yarn-toyarn distances. When the semi-interlaced fabrics were compared with the non-interlaced structures, fillingfilling and z-z yarn distances were found to be similar in satin-weave and non-interlaced orthogonal fabrics. In plain-weave fabrics, however, filling-filling and $\mathrm{z}-\mathrm{z}$ yarn distances were found to be greater than other types of fabrics. This was attributed to relatively large number of warp-filling interlacements in plain-woven fabrics which prevent the adjacent filling yarns from approaching each other during the beat-up action. The same can be stated for the z-yarns. This effect was limited in satin-woven fabrics due to a smaller number of interlacement points in satin fabrics. Hence fillingfilling and $\mathrm{z}-\mathrm{z}$ distances were similar to those of the non-interlaced fabrics. When composite properties are considered, directional fiber volume fractions in filling and $\mathrm{z}$ directions will be lower in semi-interlaced structures when compared to non-interlaced fabric composites.

Figure 11 shows the relation between the fabric type and the yarn-to-yarn distances measured on the fabric cross section (yz-plane). Filling-filling, warp-warp and $\mathrm{z}-\mathrm{z}$ distances measured on fabric cross section plane were in a narrow range of 0.2 to $0.6 \mathrm{~cm}$. As the number of layers was increased, warp-warp distance was found to decrease. Warp-warp and filling-filling distances (distances between the adjacent layers) were lower in semi-interlaced structures due to the more compact nature of the fabrics resulting from a large number of interlacements. $z-z$ distance increased as the number of layers was increased. On the other hand, z-z distances were greater in semi-interlaced structures. In these structures, warp- filling interlacement points prevent the adjacent $z$-yarns from approaching each other, thus increasing the $\mathrm{z}-\mathrm{z}$ yarn distances.

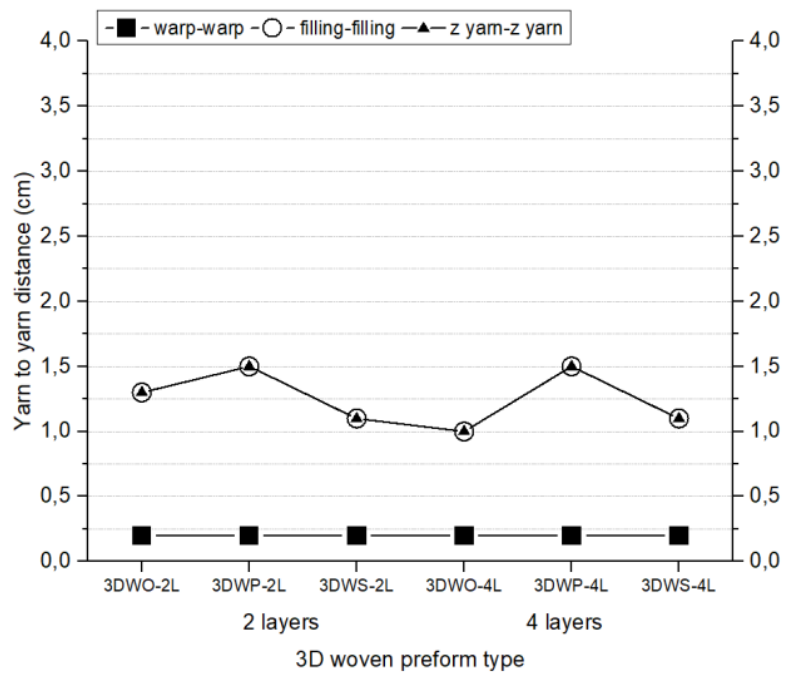

Figure 10. The relation between the fabric type and the yarn-to-yarn distances measured on the fabric side (xzplane).

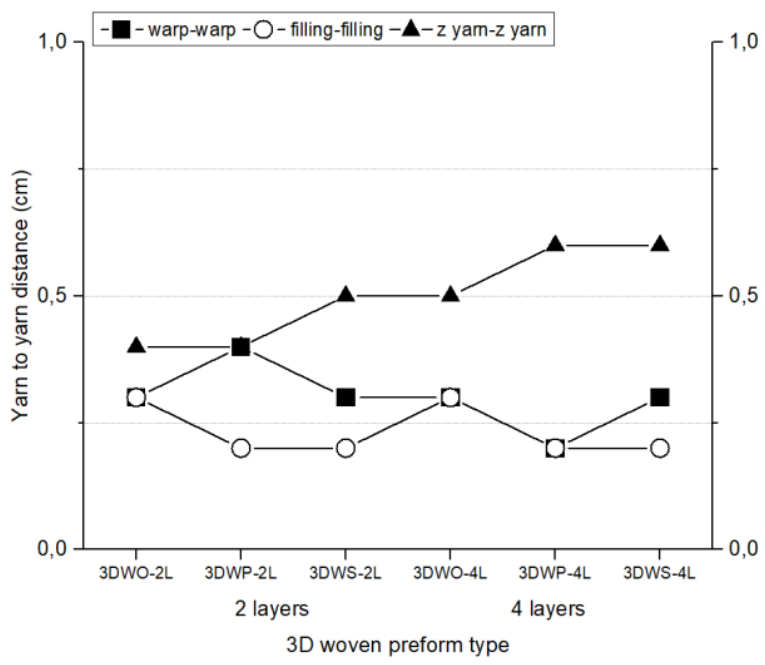

Figure 11. The relation between the fabric type and the yarn-to-yarn distances measured on the fabric cross section (yz-plane).

Figure 12 shows the relation between the fabric type and the filling and z-yarn densities measured on the 
fabric side (xz-plane). It was observed that the filling and z-yarn density values are very similar to each other. (Figure 12). Filling-filling and $\mathrm{z}-\mathrm{z}$ yarn densities were lower in plain-weave semi-interlaced fabrics in line with the increment observed in filling-filling and $\mathrm{z}-\mathrm{z}$ yarn distances in these structures. As explained above, warpfilling interlacement points in plain-weave semiinterlaced fabrics prevent the adjacent filling yarns from approaching each other, thus increasing the yarn-to-yarn distances and lowering the yarn density values. However, the effect of warp-filling interlacements was minimized in satin-weave semi-interlaced fabrics due to a lower number of warp-filling interlacements in satin structure. Accordingly, yarn density values were similar to those of the non-interlaced fabrics and higher than those of the plain-weave fabric samples.

Figure 13 shows the relation between the fabric type and the warp and z-yarn densities measured on the fabric cross-section (yz-plane). Warp yarn density values were found to be twice as higher as the z-yan densities (Figure 13). This is an expected result because one $\mathrm{z}$ yarn is placed between two adjacent warp yarns when producing 3D fabric structures. The measured yarn densities are indicative of a successful 3D fabric weaving operation which resulted in uniform fabric structures. In semi-interlaced fabrics, z-yarn densities were lower due to increasing $\mathrm{z}-\mathrm{z}$ distance. $\mathrm{z}$-directional fiber volume fraction in these structures should be expected to be lower.

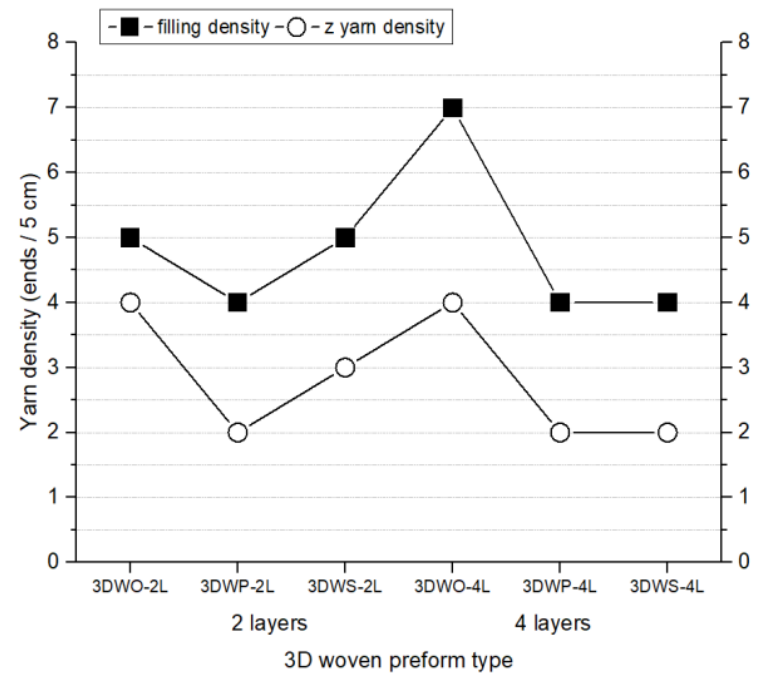

Figure 12. The relation between the fabric type and the filling and z-yarn densities measured on the fabric side (xz-plane).

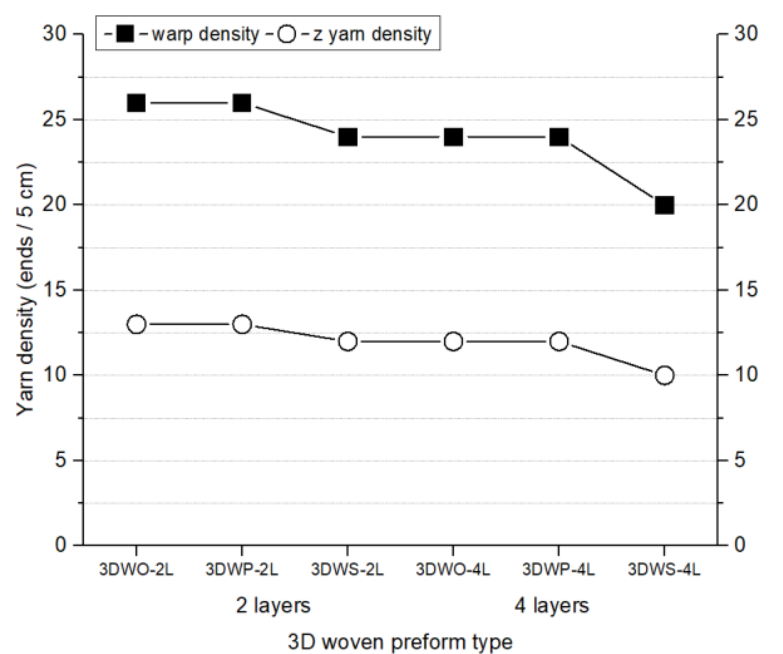

Figure 13. The relation between the fabric type and the warp and z-yarn densities measured on the fabric crosssection (yz-plane).

\subsection{Yarn Lengths}

Table 2 shows the uncrimped warp yarn length $(l w)$, filling yarn length $(l f)$, and z-yarn length $(l z)$. Figure 14 shows the relation between the fabric types and various yarn lengths. Uncrimped warp and filling yarn lengths were greater in semi-interlaced plain and satin-woven fabrics when compared to non-interlaced structures. In non-interlaced fabric structure, warp and filling yarns run along $\mathrm{x}$ and $\mathrm{y}$ axes respectively without making any interlacements with other yarn sets and preserving their straight form. However, in semi-interlaced plain and satin fabrics, warp and filling yarns make interlacements with each other according to the weave pattern and become curved. Therefore, the uncrimped warp and filling yarn lengths are greater in semi-interlaced fabrics as expected. Uncrimped z-yarn lengths were found to be greater in plain-woven fabrics compared with satinweave due possibly to an increased number of yarn interlacements which increase the yarn crimp.

Table 2. The uncrimped warp yarn length $(l w)$, filling yarn length $(l f)$, and z-yarn length $(l z)$ in $3 \mathrm{D}$ fabrics.

\begin{tabular}{lllll}
\hline $\begin{array}{l}\text { Sample } \\
\text { code }\end{array}$ & $\begin{array}{l}\text { Num } \\
\text { of } \\
\text { layers }\end{array}$ & $\begin{array}{l}l w \\
(/ 20 \mathrm{~cm})\end{array}$ & $\begin{array}{l}l f \\
(/ 9 \mathrm{~cm})\end{array}$ & $\begin{array}{l}l z \\
(\mathrm{~cm})\end{array}$ \\
\hline 3DWO & 2 & 20.0 & 9.0 & 2.1 \\
& 4 & 20.0 & 9.0 & 2.5 \\
3DWP- & 2 & 21.1 & 9.6 & 2.3 \\
ZO & 4 & 21.3 & 9.8 & 3.1 \\
3DWS- & 2 & 21.2 & 9.5 & 2.2 \\
ZO & 4 & 21.3 & 9.6 & 2.7 \\
\hline
\end{tabular}




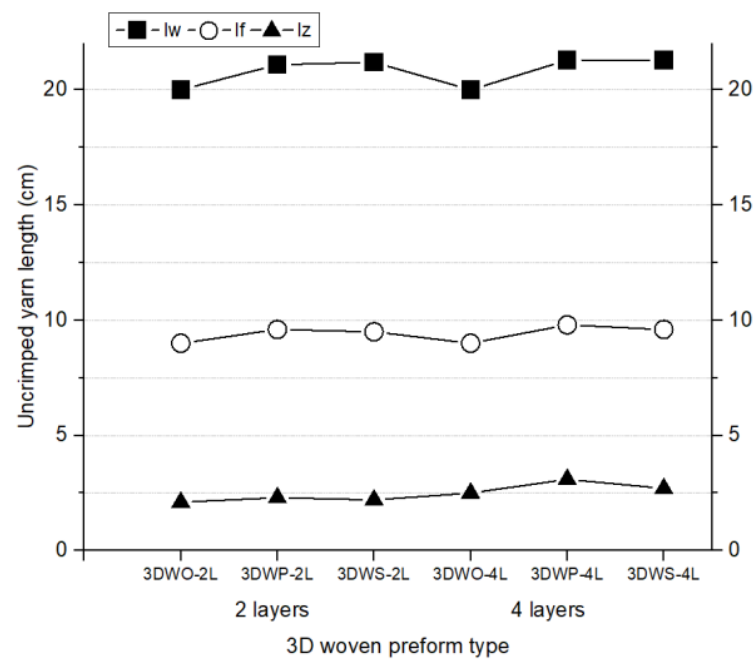

Figure 14. The relation between the fabric types and various yarn lengths.

\subsection{Yarn Angles}

Figure 15 shows the relation between the fabric type and various yarn angles. $\pm \theta z$ angle was found to be greater compared with other angles $(\theta w$ and $\theta f) . \theta z$ angle was found to be greater in non-interlaced orthogonal fabrics compared with semi-interlaced structures due to the fact that filling-filling and $\mathrm{z}-\mathrm{z}$ yarn distances are greater in semi-interlaced structures. $\theta z$ angle generally increases as the number of layers is increased. In non-interlaced fabrics, $\theta f$ and $\theta w$ angles are equal to $0^{\circ}$ due to the fact that these fabric structures, warp and filling yarns run along $\mathrm{x}$ and $\mathrm{y}$ axes respectively without making any interlacements with other yarn sets and preserving their straight form. $\theta \mathrm{w}$ angle is greater in satin woven fabrics compared with the plain weave. This was attributed to the fact that filling-filling distance measured in $\mathrm{xz}$ plane is lower in satin woven fabrics compared with the plain-weave (Figure 10). $\theta f$ angle was found to be greater in 2-layer satin fabrics when compared to 2-layer plain fabrics. However, the $\theta f$ angle was greater in the case of 4-layer plain woven fabrics when compared to satin-weave. This result is also in parallel with yarn distances measured in the yz-plane (Figure 11). Accordingly, $\theta f$ angle increases as the distance between the adjacent warp yarns measured in the yz plane decreases.

It can be concluded that $\theta f$ and $\theta w$ angles are generally affected by weave pattern whereas $\theta z$ angle is generally influenced by weaving operations such as warp let-off, multilayer filling insertion, and beat-up.

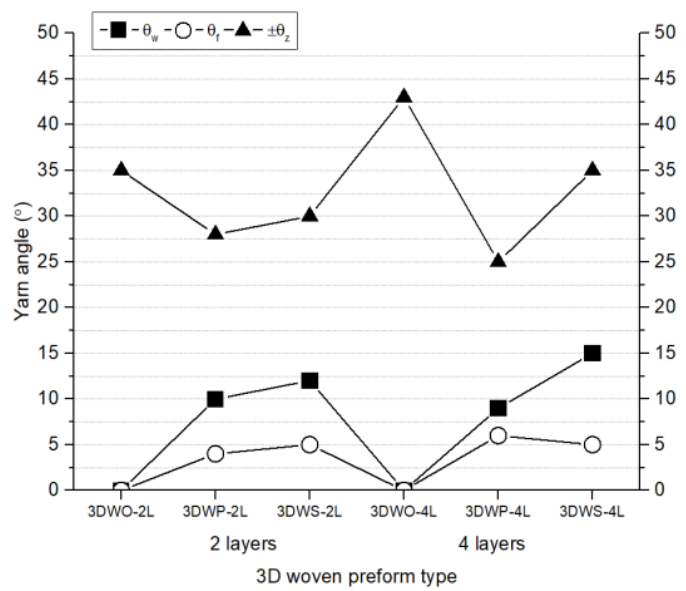

Figure 15. The relation between the fabric type and various yarn angles.

\section{Conclusion}

Mechanical properties of a composite material are dependent upon the fiber type, matrix type, fiber orientation/architecture, and fiber volume fraction. For 3D textile reinforced composites, the weave pattern, number of layers, yarn angles and other parameters are the main factors determining the fiber orientation and architecture and hence the directional and overall fiber volume fractions. In this study, geometric characterization of $3 \mathrm{D}$ woven jute fabrics was carried out. For this purpose, two main types of 3D fabrics were produced such as non-interlaced and semi-interlaced fabrics. The semi-interlaced fabrics were produced using two different weave patterns such as plain and satin weave. Two different numbers of layers i.e. 2 and 4 layers were used for all types of fabrics produced in the study.

Yarn-to-yarn distances and yarn densities: Increasing the number of layers did not have a significant effect on yarn-to-yarn distances. Filling-filling and $\mathrm{z}-\mathrm{z}$ yarn distances were found to be greater in plain-woven semiinterlaced fabrics when compared to satin-weave semiinterlaced fabrics and non-interlaced orthogonal fabrics due to a large number of interlacements in plain-weave structure. It can be expected that the filling and $\mathrm{z}$ directional fiber volume fractions would be lower in semi-interlaced fabric reinforced composite structures. Yarn density values were also found to be in parallel with the yarn-yarn distance results.

Yarn lengths: The uncrimped warp and filling yarn lengths were greater in semi-interlaced plain and satin fabrics compared with those of non-interlaced structures. Uncrimped z-yarn lengths were greater in plain-woven fabrics when compared to the satin-weave due to larger number of yarn interlacements and hence the yarn curviness in plain-weave. 
Yarn angles: $\mathrm{z}$ yarn angle was found to be greater than other angles. $\mathrm{z}$ yarn angle was greater in non-interlaced fabrics compared with semi-interlaced fabrics due to the fact that filling-filling and $\mathrm{z}-\mathrm{z}$ yarn distances were greater in semi-interlaced fabrics. $z$ yarn angle increased as the number of layers was increased for all fabric types. Warp yarn angle was greater in satin fabrics compared to plain-woven structures. Filling and warp angles are generally affected by weave pattern whereas $\mathrm{z}$ yarn angle is influenced by weaving operations such as warp let-off, multilayer filling insertion, and beat-up. The findings of this study will shed light on the effect of $3 \mathrm{D}$ fabric type and geometry on the properties of natural fiber reinforced 3D woven composites.

\section{Author's Contributions}

Nesrin Şahbaz Karaduman: Produced the samples, carried out the measurements, performed the experiments and analyzed the results, drafted and wrote the manuscript.

\section{Ethics}

There are no ethical issues after the publication of this manuscript.

\section{References}

1. Stig, F, Hallström, S. 2012. Spatial modelling of 3D-woven textiles. Composite Structures; 94(5): 1495-1502.

2. Ko, F. 3-D textile reinforcements in composite materials. In: Miravete A (ed) 3-D textile reinforcements in composite materials. Woodhead Publishing, Cambridge, 1999, pp 9-42.

3. Mohanty, AK, Misra, M, Drzal, LT. Natural fibers, biopolymers, and biocomposites: An introduction. In: Mohanty A, Misra M, Drzal LT (eds) Natural fiber, biopolymer, and biocomposites. CRC Press, Boca Raton, 2005, pp 1-36.

4. Aziz, SH, Ansell, MP. Optimising the properties of green composites. In: Baillie C (ed) Green composites: Polymer composites and the environment. Woodhead Publishing, Boca Raton, 2004, pp 154-180.

5. Satyanarayana, KG, Arizaga, GGC, Wypych, F. 2009. Biodegradable composites based on lignocellulosic fibers-An overview. Progress in Polymer Science; 34(9): 982-1021.

6. Mohanty, AK, Misra, M, Hinrichsen, G. 2000. Biofibres, biodegradable polymers and biocomposites: An overview. Macromolecular Materials and Engineering; 276-277(1): 1-24.

7. Bismarck, A, Mishra, S, Lampke, T. Plant fibers as reinforcement for green composites. In: Mohanty A, Misra M, Drzal LT (eds) Natural fibers, biopolymers, and biocomposites. CRC Press, Boca Raton, 2005, pp 37-108.

8. Wambua, P, Ivens, J, Verpoest, I. 2003. Natural fibres: Can they replace glass in fibre reinforced plastics? Composites Science and Technology; 63(9): 1259-1264.

9. Joshi, SV, Drzal, LT, Mohanty, AK, Arora, S. 2004. Are natural fiber composites environmentally superior to glass fiber reinforced composites? Composites Part A: Applied Science and Manufacturing; 35(3): 371-376.
10. Mehta, G, Mohanty, AK, Thayer, K, Misra, M, Drzal, LT. 2005. Novel biocomposites sheet molding compounds for low cost housing panel applications. Journal of Polymers and the Environment; 13: 169-175.

11. Bledzki, AK, Faruk, O, Sperber, VE. Cars from bio-fibres. 2006. Macromolecular Materials and Engineering; 291(5): 449-457.

12. Bilisik, K, Karaduman, NS, Bilisik, NE, Bilisik, HE. 2013. Threedimensional fully interlaced woven preforms for composites. Textile Research Journal; 83(19): 2060-2084.

13. Potluri, P, Sharif, T, Jetavat, D. 2008. Robotic approach to textile preforming for composites. Indian Journal of Fibre \& Textile Research; 33: 333-338. 